\title{
FEM of Gas-Assisted Injection Molding Based on 3D Model
}

\author{
Xinchao Wang $\mathbb{D},{ }^{1,2}$ Tie Geng $\mathbb{D},{ }^{1}$ Liqun Yan, ${ }^{1}$ Yonggang Guo, ${ }^{1}$ and Lih-Sheng Turng ${ }^{2,3}$ \\ ${ }^{1}$ School of Mechatronic Engineering, and National Engineering Laboratory for Wheat \& Corn Further Processing, \\ HeNan University of Technology, Zhengzhou 450001, China \\ ${ }^{2}$ Wisconsin Institute for Discovery, University of WI-Madison, Madison, WI, USA \\ ${ }^{3}$ Department of Mechanical Engineering, University of WI-Madison, Madison, WI, USA \\ Correspondence should be addressed to Tie Geng; tiegeng2000@163.com
}

Received 29 July 2019; Revised 18 November 2019; Accepted 3 December 2019; Published 30 March 2020

Academic Editor: Gyorgy Szekely

Copyright ( 12020 Xinchao Wang et al. This is an open access article distributed under the Creative Commons Attribution License, which permits unrestricted use, distribution, and reproduction in any medium, provided the original work is properly cited.

\begin{abstract}
The gas-assisted injection molding (GAIM) process is so complicated that increasing reliance has been placed on CAE (Computer Aided Engineering) as a tool for both mold designers and process engineers. In this paper, a 3D theoretical model and numerical scheme is presented to simulate the GAIM process, in which an equal-order velocity-pressure formulation method is employed to eliminate the pressure oscillation. In addition, the whole flow field including the gas and melt regions is calculated using a uniform momentum equation with the viscosity of gas raised to a certain order of magnitude, and a $3 \mathrm{D}$ control volume scheme is employed to track the flow front of the melt and gas. Finally, the validity of the model has been tested through case studies and experimental verification.
\end{abstract}

\section{Introduction}

Gas-assisted injection molding (GAIM) is one of the important and innovative molding processes and considered to be a revolution in terms of injection molding technology $[1,2]$. This new process came into practice as ripe technology in the 1990s, and has been spread widely due to its outstanding advantages. Compared with conventional injection molding (CIM), GAIM offers a considerable number of advantages, such as reduced part weight, injection pressure, clamp force, shrinkage, warpage, and residual stress. GAIM has four types: standard molding, vice cavity molding, melt backflow, and core activities [1]. This paper mainly focuses on standard molding, which is also called partial filling and inflation.

In gas-assisted injection molding, the mold cavity is partially, about $70-90 \%$ of the mold cavity, filled with the polymer melt, and then the gas with high pressure will be injected into the melt immediately or after some delay time. Three distinct regions can be indentified during the gas-assisted injection molding filling stage (as shown in Figure 1): the solidified melt layer close to the mold wall, the deforming viscous melt, and the penetration gas. The three regions are confined by the melt and gas fronts. In this process, the melt filling process in GAIM is the same as that in CIM, and the penetration of gas into melt will force the melt to fill the whole mold cavity.

But, with the gas injection, the GAIM process becomes so complicated that at present, the understanding of the characteristics of the process is still lagging behind.

In GAIM, two dramatically dissimilar materials flowing within complex cavities interact dynamically, and many additional parameters are introduced, such as the numbers and location of gas injection points, the short-shot size, gas delay time, the injected gas pressure, and the holding time for gas injection. So, the molding design and process control become more critical and difficult than conventional injection molding. Furthermore, previous experience about conventional injection molding is no longer sufficient to deal with this process.

So, the computer simulation for gas-assisted injection molding is needed urgently to improve the mold design and process control. Now, increasing reliance has been placed on computer-aided engineering (CAE), and commercially available computer simulation packages for the injection molding process have become routine tool for both mold 


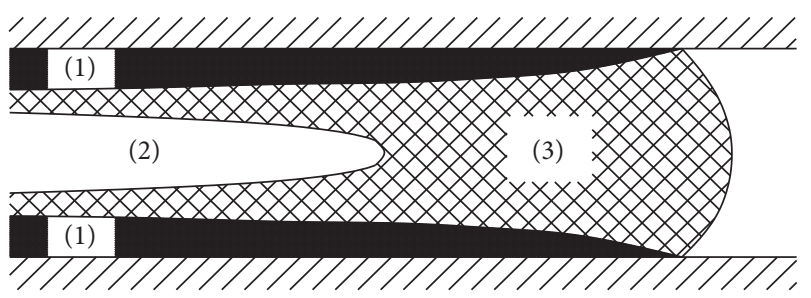

Figure 1: Schematic of gas-assisted injection molding.

designers and process engineers. With CAE simulation tools, engineers can optimize the mold and products, which used to be conducted by the trial-and-error method that is expensive and inefficient.

During the past decades, most of the papers on the simulation of GAIM are based on either a midplane model [3] or a surface model [4] which is both a so-called 2.5D approach. In the $2.5 \mathrm{D}$ method, the melt filling in the thin cavity is assumed as Hele-Shaw flow [5], and the velocity and the variation of pressure in the gapwise direction are neglected except that the temperature is solved by FDM, so the filling of a mold cavity becomes a $2 \mathrm{D}$ problem in flow direction and a $1 \mathrm{D}$ problem in gapwise direction. The residual wall thickness of a part is used to represent the gas penetration, which is determined by capillary number or empirical formula [6]. The influence of gas penetration on melt flow is taken only as a boundary condition of the melt filling region.

The models based on Hele-Shaw are simple and have less time cost, but they are used mainly for the analysis of shell structure parts without 3D features. As for these molded parts with the more complex three-dimensional geometries and uniform thickness walls often encountered in gasassisted injection molding, the velocity and the changes of parameters in the gapwise direction are considerable and cannot be neglected. As for GAIM, the information obtained by 2.5 -dimensional models is often limited and incomplete. Several phenomena occurring during mold filling, such as the fountain effect and fiber orientation, cannot be accurately predicted using the Hele-Shaw approximation. In many cases, the calculation results generated by the $2.5 \mathrm{D}$ model cannot coincide with experimental results [7].

At present, there are not many research papers on the simulation of three-dimensional GAIM. Literature [8] focuses on the $3 \mathrm{D}$ numerical simulation of the moving interfaces in GAIM using the CLSVOF method. Although several GAIM simulation commercial softwares, such as MOLDFLOW, MOLDEX3D, etc. [9, 10], have been developed, which have both three-dimensional analysis module of GAIM, their research results are regarded as the company's core technology and are strictly confidential, and the published articles on it are limited to the introduction of major functions, and the technical details are not disclosed to the public.

In this paper, a 3D finite element model is presented to deal with the melt filling, in which an equal-order velocitypressure formulation method [11] for 3D flow field is employed to eliminate the pressure oscillation. The whole flow field including the gas and melt regions is calculated using a uniform momentum equation with the viscosity of gas raised to a certain order of magnitude. A 3D control volume scheme is employed to track the flow front of the melt and gas. The software based on this 3D model has been developed and can calculate the pressure field, velocity field, temperature field, and gas penetration in the injection process without pressure oscillation. The validity of the model has been tested through case studies and experimental verification.

\section{Mathematical Model}

Due to the absence of the Hele-Shaw approximation and the dynamic interaction of the gas and melt, the GAIM process becomes more complicated. Hence, several assumptions are needed and are listed below.

(1) The pressure of the melt is not very high during filling of the cavity; hence, the melt is considered incompressible and purely viscous.

(2) The polymer melt has high viscosity, so inertia, gravitation, and surface tension of melt are neglected as compared with the viscous force.

(3) The gas is treated as the incompressible fluid.

(4) Temperature in the gas regions is considered same everywhere and permanent. Furthermore, there is no thermal exchange between the melt and gas.

Given the abovementioned approximations, the governing equations, expressed in Cartesian coordinates, are as follows.

Momentum equations:

$$
\begin{aligned}
\frac{\partial}{\partial x}\left(2 \eta \frac{\partial u}{\partial x}\right)+\frac{\partial}{\partial y}\left[\eta\left(\frac{\partial v}{\partial x}+\frac{\partial u}{\partial y}\right)\right]+\frac{\partial}{\partial z}\left[\eta\left(\frac{\partial w}{\partial x}+\frac{\partial u}{\partial z}\right)\right] \\
-\frac{\partial(P)}{\partial x}=0
\end{aligned}
$$

$$
\begin{aligned}
\frac{\partial}{\partial x} & {\left[\eta\left(\frac{\partial v}{\partial x}+\frac{\partial u}{\partial y}\right)\right]+\frac{\partial}{\partial y}\left(2 \eta \frac{\partial v}{\partial y}\right)+\frac{\partial}{\partial z}\left[\eta\left(\frac{\partial w}{\partial y}+\frac{\partial v}{\partial z}\right)\right] } \\
& -\frac{\partial(P)}{\partial y}=0
\end{aligned}
$$

$$
\begin{aligned}
& \frac{\partial}{\partial x}\left[\eta\left(\frac{\partial w}{\partial x}+\frac{\partial u}{\partial z}\right)\right]+\frac{\partial}{\partial y}\left[\eta\left(\frac{\partial v}{\partial z}+\frac{\partial w}{\partial y}\right)\right]+\frac{\partial}{\partial z}\left(2 \eta \frac{\partial w}{\partial z}\right) \\
& -\frac{\partial(P)}{\partial z}=0 .
\end{aligned}
$$

Continuity equation:

$$
\frac{\partial u}{\partial x}+\frac{\partial v}{\partial y}+\frac{\partial w}{\partial z}=0
$$

Energy equation: 


$$
\begin{aligned}
\rho C_{P} \frac{\partial T}{\partial t}= & \rho C_{P}\left(u \frac{\partial T}{\partial x}+v \frac{\partial T}{\partial y}+w \frac{\partial T}{\partial z}\right) \\
& +\frac{\partial}{\partial x}\left(K \frac{\partial T}{\partial x}\right)+\frac{\partial}{\partial y}\left(K \frac{\partial T}{\partial y}\right)+\frac{\partial}{\partial z}\left(K \frac{\partial T}{\partial z}\right)+\eta \dot{\gamma}^{2}
\end{aligned}
$$

where $x, y, z$ are the three-dimensional coordinates and $u, v, w$ are the velocity components in the $x, y, z$ directions, respectively. $P, T, \rho$, and $\eta$ denote the pressure, temperature, density, and viscosity, respectively.

The cross viscosity model has been used for the simulations:

$$
\eta=\frac{\eta_{0}(T, P)}{1+\left(\eta_{0} \dot{\gamma} / \tau^{*}\right)^{1-n}} .
$$

Because there is no notable change in the scope of the temperature of the polymer melt during filling, the Arrhenius model [12] for $\eta_{0}$ is employed as follows.

$$
\eta_{0}(T, P)=B \exp \left(\frac{T_{b}}{T}\right) \exp (\beta P) .
$$

\section{Numerical Implementation}

3.1. Melt Injection. For the 3D simulation of injection molding, the main difficulty is pressure oscillations, i.e., it is hard to obtain a stable solution during calculating the pressure and velocity. To solve this problem, an equal-order velocitypressure formulation method is given here which uses 4-node tetrahedron elements based on FEM [13]. The main idea of this method is (1) the relationship between velocity and pressure is obtained from the discretized momentum equations, and then the pressure equation is obtained by substituting the velocity expressions into discretized continuity equation. (2) After pressure calculation, the velocity needs to be updated. (3) The overall calculation process is iterative.

The momentum equations are discretized using Galerkin's method with equal-order velocity-pressure formulation. The element equations are assembled in the conventional manner to form the discretized global momentum equations, and the nodal velocity may be expressed as following:

$$
\begin{gathered}
u_{i}=\widehat{u}_{i}-K_{i}^{u} \frac{\partial P}{\partial x}, \\
v_{i}=\widehat{v}_{i}-K_{i}^{v} \frac{\partial P}{\partial y}, \\
w_{i}=\widehat{w}_{i}-K_{i}^{w} \frac{\partial P}{\partial z},
\end{gathered}
$$

where $i$ means the global node number and $\widehat{u}_{i}, \widehat{v}_{i}, \widehat{w}_{i}$, called virtual velocity of node $i$, are dependent on the velocity and velocity coefficients of the nodes around node $i . K_{i}^{u}, K_{i}^{v}, K_{i}^{w}$ denote the nodal pressure coefficients in the direction of $x, y, z$ coordinate, respectively.

Substitution of the velocity expressions (4) into the continuity equation, which is discretized using the
Galerkin method, yields the following element equation for pressure:

$$
\begin{gathered}
\int_{V}\left[\frac{\partial N_{i}}{\partial x}\left(N_{j} K_{j}^{u} \frac{\partial N_{k}}{\partial x} P_{k}\right)+\frac{\partial N_{i}}{\partial y}\left(N_{j} K_{j}^{\nu} \frac{\partial N_{k}}{\partial y} P_{k}\right)\right. \\
\left.+\frac{\partial N_{i}}{\partial z}\left(N_{j} K_{j}^{w} \frac{\partial N_{k}}{\partial z} P_{k}\right)\right] d V \\
=\int_{V}\left(\frac{\partial N_{i}}{\partial x} N_{j} \widehat{u}_{j}+\frac{\partial N_{i}}{\partial y} N_{j} \widehat{v}_{j}+\frac{\partial N_{i}}{\partial z} N_{j} \widehat{w}_{j}\right) d V,
\end{gathered}
$$

where $N$ represents the element-based interpolation function. The element pressure equations are assembled in the conventional manner to form the global pressure equations.

Because the velocity field obtained by solving momentum equations does not satisfy continuity equation, the velocity values need be updated after the pressure field has been obtained from equation (5), using the following relations:

$$
\begin{aligned}
& u_{i}=\widehat{u}_{i}-\frac{1}{A_{i i}^{x}} \int_{V} N \frac{\partial P}{\partial x} \mathrm{~d} V, \\
& v_{i}=\widehat{v}_{i}-\frac{1}{B_{i i}^{y}} \int_{V} N \frac{\partial P}{\partial y} \mathrm{~d} V, \\
& w_{i}=\widehat{w}_{i}-\frac{1}{C_{i i}^{z}} \int_{V} N \frac{\partial P}{\partial z} \mathrm{~d} V,
\end{aligned}
$$

where $A_{i i}^{x}, B_{i i}^{y}, C_{i i}^{z}$ denote the nodal velocity coefficients in the direction of $x, y, z$ coordinate, respectively.

3.2. Calculation of the Temperature Field. The temperature field plays an important role during the injection molding process. Because the viscosity of the polymer varies with its temperature, the variation of the temperature of polymer will have important influence to the injection molding process. Only after the temperature field during filling has been calculated exactly, the simulations for packing and cooling are meaningful. This paper gives a 3D model for calculating the temperature field which considers the influence of convection items on three dimensions and suitable for the wider range of parts and has more exact results compared with the $2.5 \mathrm{D}$ models. According to the energy equation (1e), by the use of Galerkin's method, the equation for the temperature field can be expressed as follows:

$$
\begin{aligned}
\int_{V} N \rho C_{p} \frac{\partial T}{\partial t} \mathrm{~d} V= & \int_{V} N\left[-\rho C_{p}\left(u \frac{\partial T}{\partial x}+v \frac{\partial T}{\partial y}+w \frac{\partial T}{\partial z}\right)\right. \\
& +\frac{\partial}{\partial x}\left(K \frac{\partial T}{\partial x}\right)+\frac{\partial}{\partial y}\left(K \frac{\partial T}{\partial y}\right) \\
& \left.+\frac{\partial}{\partial z}\left(K \frac{\partial T}{\partial z}\right)+\eta \dot{\gamma}^{2}\right] \mathrm{d} V c
\end{aligned}
$$




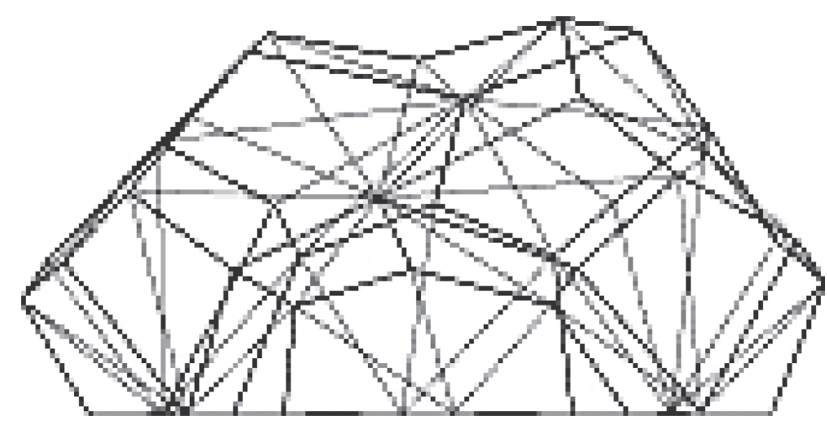

(a)

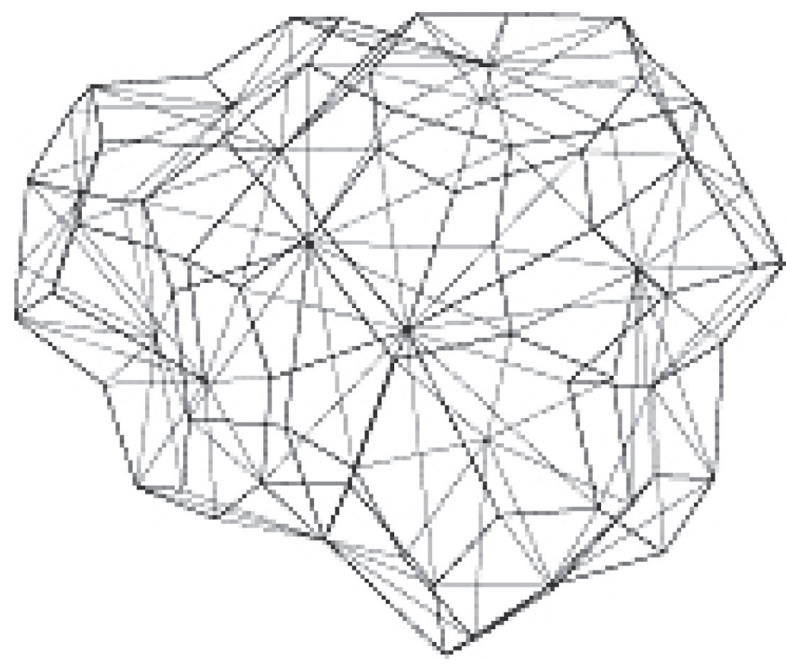

(b)

Figure 2: 3D control volumes. (a) Control volume of an internal node. (b) Control volume of a boundary node.

Thermal convection item and viscous heat item are anisotropic and has to do with the direction of flow. To keep the numerical stability, the upwind method is employed to handle the convection item and viscous heat item, e.g., only the contributions of the upriver elements from the nodes are considered when the convection item and viscous heat item are calculated. In the abovementioned equations, the time $T$ is discretized using a forward-difference method:

$$
\frac{\partial T_{j}}{\partial t}=\frac{T_{j}^{n+1}-T_{j}^{n}}{\Delta t}
$$

where $\Delta t$ denotes time step.

The element temperature equations are assembled in the conventional manner to form the global temperature equations. The overall procedure for temperature calculations is iterative. Because the pressure, velocity, and temperature influence each other during the calculation, the temperature and pressure are coupled during the procedure.

3.3. Gas Penetration. The pressure value of the gas gate node is equal to high-pressure gas pressure, and the values of temperature, viscosity, and pressure of other nodes have been gained at the end of the plastic melt filling stage, and the velocity of each node should be zero. These are the initial conditions when calculating the gas penetration and melt flow at the gas injection stage.

Here, the viscosity of gas is raised to a certain order of magnitude so that the gas and melt regions can be calculated with a uniform momentum equation although the viscosity of gas is much lower than that of polymer in fact. The adjacent nodes of the gas gate node are called the gas frontier nodes. The calculation of gas penetration is similar to calculating melt filling.

The filling time step is selected to ensure that only one node control volume is filled or replaced by gas at each time step to determine the new flow front of melt or gas. For this reason, according to the current pressure field, for melt flow, the flow rates per unit time to the melt front nodes are calculated, then the time taken for filling the first front node is determined; for gas penetration, the gas volume per unit time to the gas frontier nodes are calculated, and the time used by the gas frontier node that was first blown off is calculated. Finally, the shortest filling or blowing time is taken as the time step. The point is that the volume of the melt flowing out is equal to that of the gas penetration at each time step.

3.4. Trace of the Flow Fronts. The flow of plastic melt in the cavity and the penetration of gas in the melt are unsteady, and the positions of the flow fronts vary with time. Like in the $2.5 \mathrm{D}$ model, in this paper, the control volume method is employed to trace the position of the flow fronts of the melt and gas after the FAN (flow analysis network) [14]. But, 3D control volume is a special volume and more complex than the $2 \mathrm{D}$ control volume. It is required that $3 \mathrm{D}$ control volumes of all nodes fill the cavity without gap and hollow space. Two kinds of 3D control volumes are shown in Figure 2. The detailed description of the 3D control volume can be found in reference [13].

\section{Verification}

4.1. Experimental Product and Mould. An experimental cavity was employed for the purpose of verification. The experimental product was $200 \mathrm{~mm}$ long, with the crosssectional shape, and the dimensions of the product are presented in Figure 3.

The mould used in this experiment was made by JiaRen Mould Company of Zhejiang province, China, shown as in Figure 4. PP Yuplene R370Y from SK Corporation, which is a common and representative material of GAIM [15], was selected as the simulative and experimental material. The short-shot size was $85 \%$, injection melt temperature was $230^{\circ} \mathrm{C}$, mould temperature was $40^{\circ} \mathrm{C}$, gas pressure was $10 \mathrm{MPa}$, and gas injection time was $10 \mathrm{~s}$. 


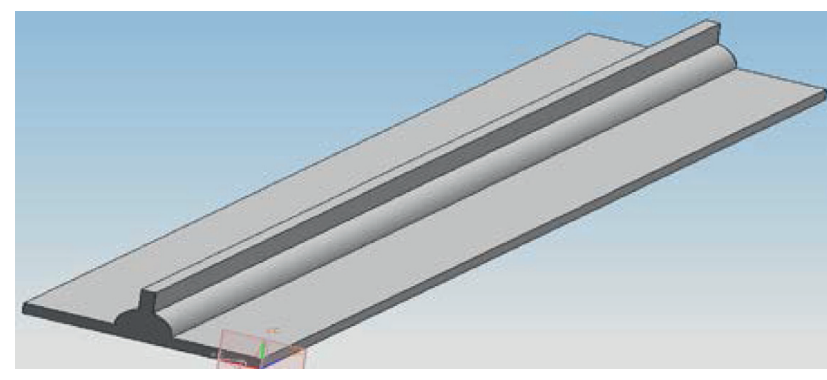

(a)

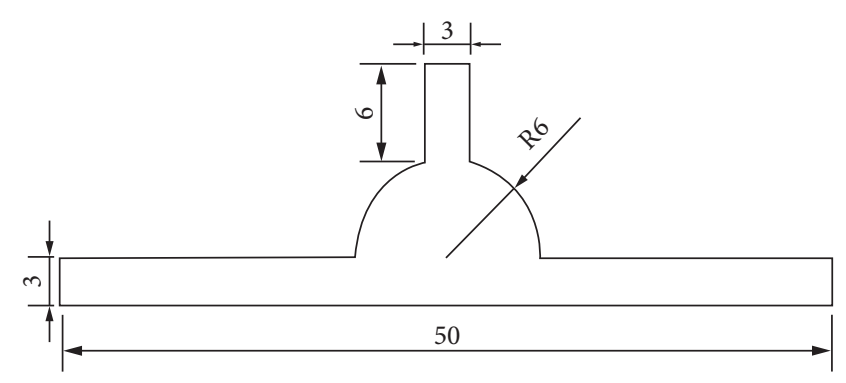

(b)

FIgURE 3: Shape and dimensions of the product.

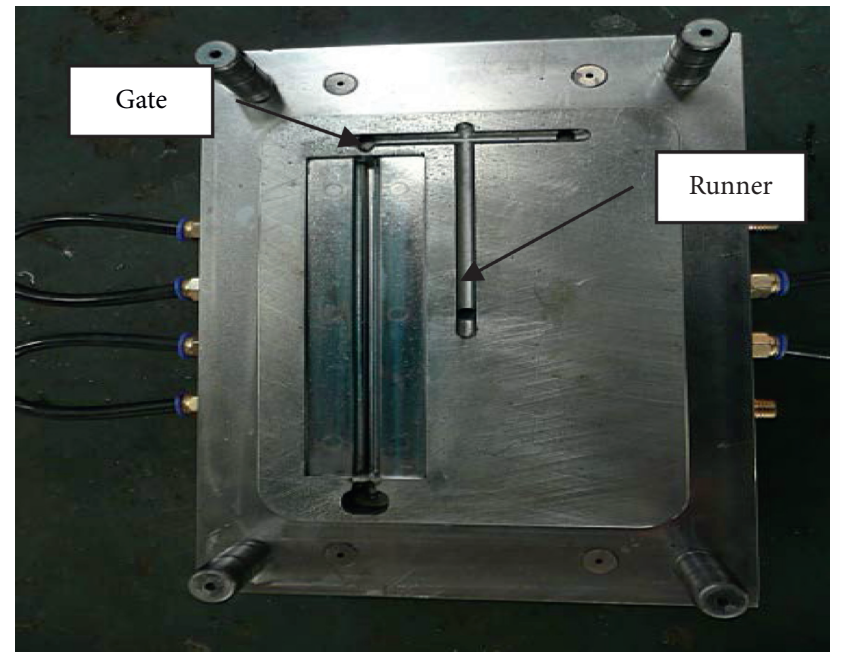

(a)

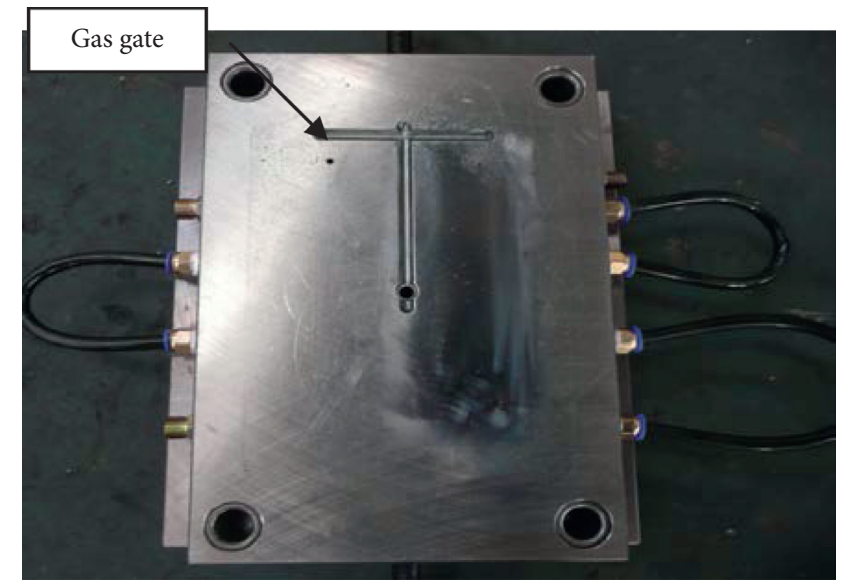

(b)

FIGURE 4: Experimental moulds.
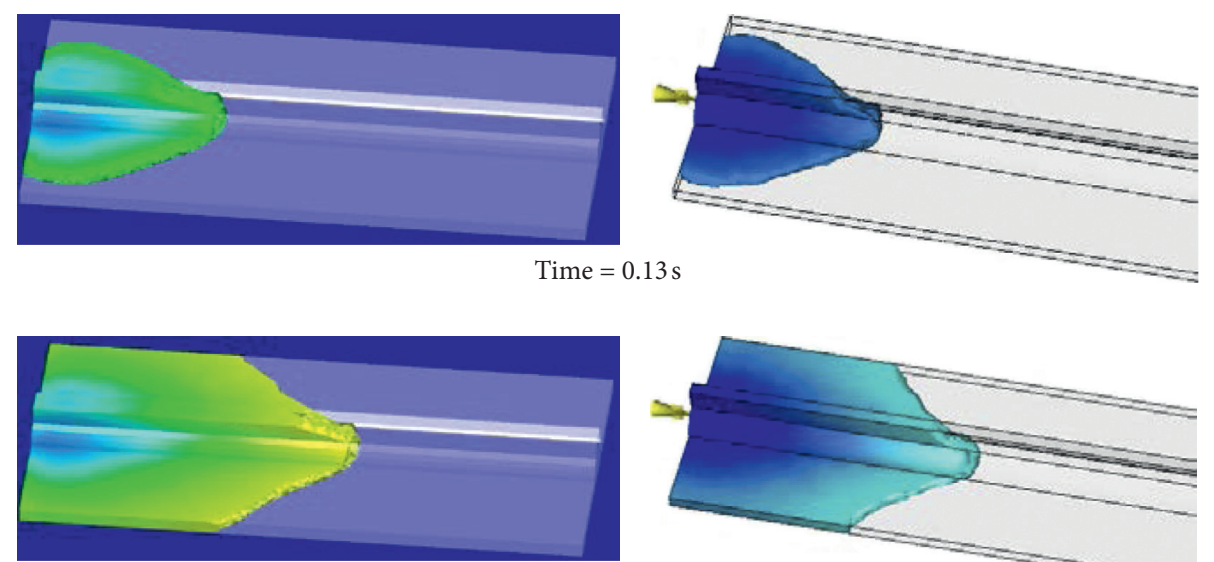

Time $=0.22 \mathrm{~s}$
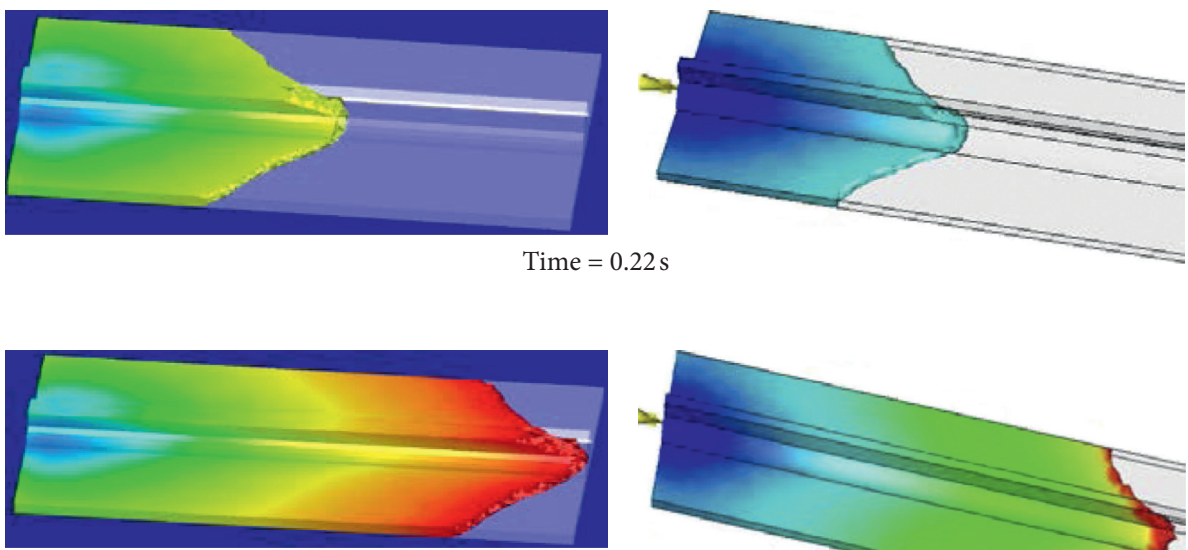

Time $=0.32 \mathrm{~s}$

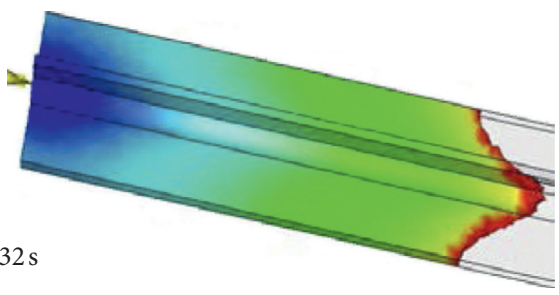

(a)

(b)

FIGURE 5: Comparisons of the flow fronts predicted by (a) this model and (b) the commercial simulation software. 


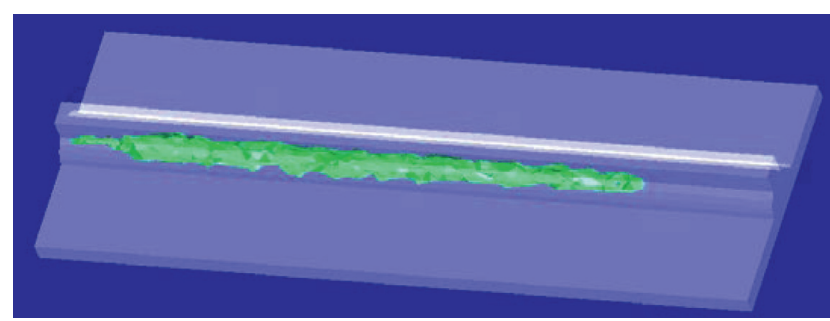

(a)

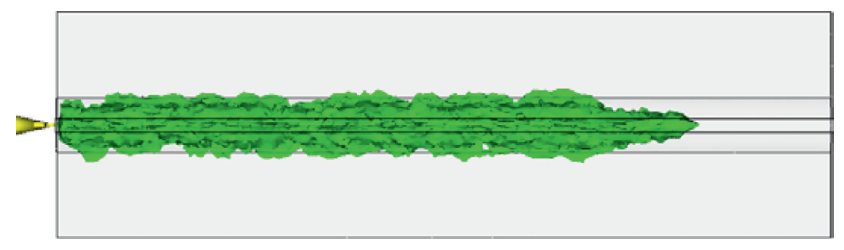

(b)

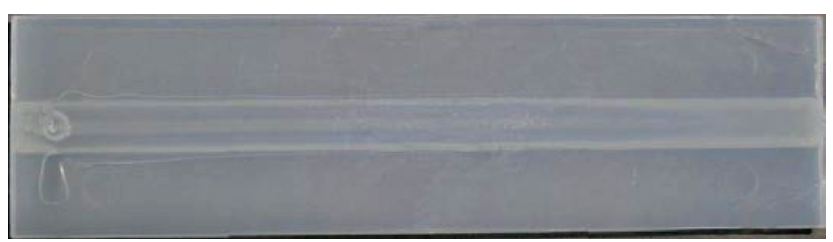

(c)

FiguRE 6: Comparisons among predicted gas penetration length by this model, the commercial simulation software, and experimental observation. (a) Result by this model. (b) Result by the commercial software. (c) Experimental result.

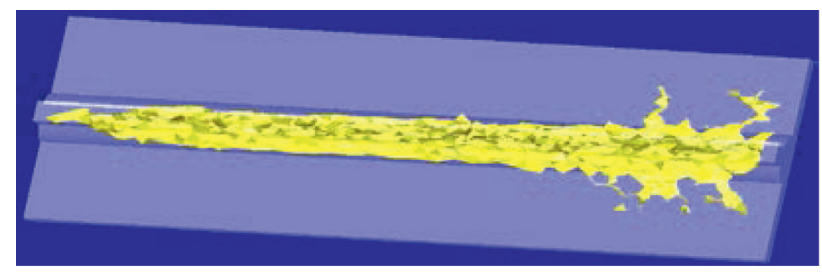

(a)

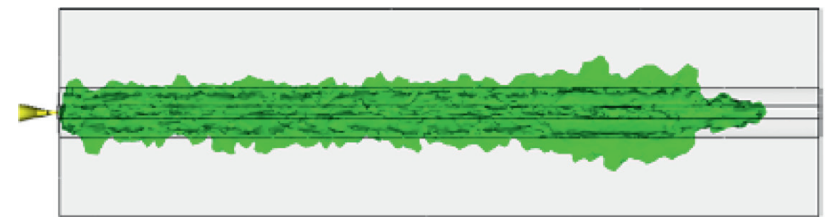

(b)

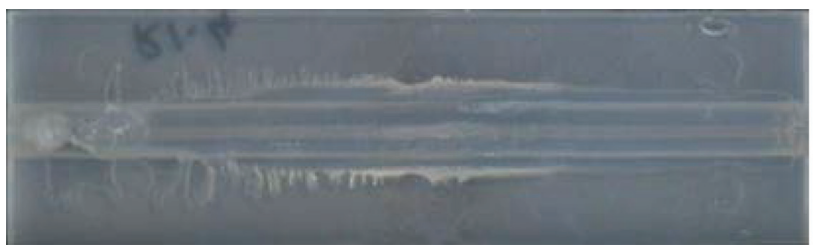

(c)
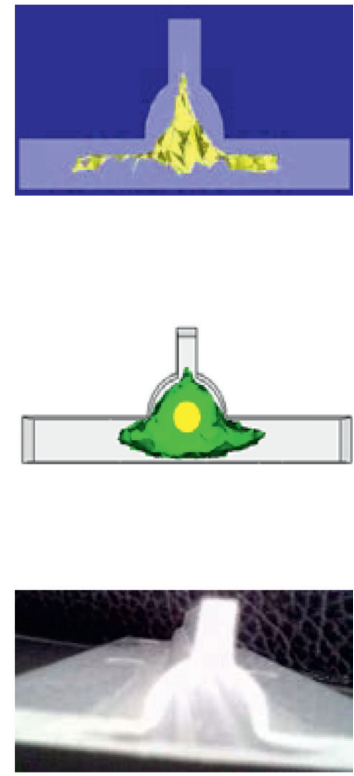

FIGURE 7: Comparisons among predicted fingering width range by this model, the commercial simulation software, and experimental observation. (a) Predicted result by this model. (b) Predicted result by commercial simulation software. (c) Experimental result.

4.2. Experimental Results and Discussion. In addition, the predicted result by this model was compared with that of certain a commercial simulation software to verify this model. The commercial software used was the Moldflow 2018 version in this study. The flow fronts of the melt at 3 different time steps simulated by this model and the commercial simulation software are shown in Figure 5. It can be seen that the injection sequence and the flow fronts of the melt at the same time step during the injection process predicted by the present model is in good agreement with that predicted by the commercial simulation software.
The comparison among predicted gas penetration length at certain gas injection time by this model, the commercial simulation software, and experimental observations is illustrated in Figure 6. It can be seen that the predicted direction and length of gas penetration based on this model agree well with that based on the commercial simulation software and also agree well with the experimental observation.

Another comparison among predicted fingering width range by this model, the commercial simulation software, and experimental observation is illustrated in Figure 7. It can 
be seen that the maximum fingering width range predicted by both this model and the commercial simulation software are basically consistent with the experimental result. However, the cross-sectional shape of the gas channel predicted by the commercial simulation software agrees better with the experimental observation than that predicted by the present model.

\section{Conclusions}

A mathematic model and numerical algorithms to simulate the gas-assisted injection molding based on a 3D finite element method is presented in this paper. The model uses the equal-order velocity-pressure formulation method to prevent the potential numerical instabilities. The whole flow field including the gas and melt regions is calculated using a uniform momentum equation with the viscosity of gas raised to a certain order of magnitude. Also, a 3D control volume scheme is adopted to track the flow front of the melt and gas. The experimental verification and comparison show that the present model is valid and reliable.

\section{Data Availability}

The datasets used to support the findings of this study are available from the corresponding author upon request.

\section{Conflicts of Interest}

The authors declare that they have no conflicts of interest.

\section{Acknowledgments}

The authors would like to acknowledge financial support from the National Natural Science Foundation of China (Grant no. 51375143), the Henan Provincial Education Department Science and Technology Project (Grant no. 19A460016, and 18A430010), the Henan Provincial Science and Technology Project (Grant no. 182102210087), the Open Research Subject of National Engineering Laboratory for Wheat \& Corn Further Processing (Grant no. NL201613), and Talent Support Fund of Henan University of Technology (Grant no. 2017QNJH25 and 216BS026).

\section{References}

[1] J. Avery, Gas-Assist Injection Molding Principles and Applications, Chemical Industry Press, Shanghai, China, 2003.

[2] S. Liang, N. Sun, and W. Yang, "The MPI 3D-simulation and experiment of the gas penetration behavior during gasassisted injection molding," Polymer Materials Science and Engineering, vol. 25, no. 8, pp. 877-892, 2009.

[3] H. Zhou and D. Li, "Filling simulation and gas penetration modeling for gas-assisted injection molding," Applied Mathematical Modelling, vol. 27, no. 11, pp. 849-860, 2003.

[4] J. Li, L. Chen, H. Zhou, and D. Q. Li, "Surface model based modeling and simulation of filling process in gas-assisted injection molding," Journal of Manufacturing Science and Engineering, vol. 131, pp. 262-274, 2009.

[5] C. A. Hiebert and S. F. Shen, "A finite-element/finite-difference simulation of injection molding filling process,"
Journal of Non-Newtonian Fluid Mechanics, vol. 7, no. 1, pp. 152-164, 1980.

[6] R. A. Sousa, R. L. Reis, A. M. Cunha, and M. J. Bevis, "Structural development of HDPE in injection molding," Journal of Applied Polymer Science, vol. 89, no. 8, pp. 20792087, 2003.

[7] Y. Zhou, B. Dong, and J. B. Chen, "Numerical simulation of the filling stage in gas-assisted injection molding and contrast to experiment," Polymer Materials Science and Engineering, vol. 22, no. 5, pp. 28-31, 2006.

[8] Q. Li, J. Ouyang, B. Yang, and X. Li, "Numerical simulation of gas-assisted injection molding using CLSVOF method," Applied Mathematical Modelling, vol. 36, no. 5, pp. 2262-2274, 2012.

[9] C. Huang and J. F. Huang, Moldflow Simulation of Injection Molding from Entry to Mastery, Machinery Industry Press, South Norwalk, CT, USA, 2017.

[10] P. Thakre, A. S. Chauhan, E. Raj Kumar, E. R. Kumar, and R. Pradyumna, "Estimation of shrinkage \& distortion in WaxInjection using Moldex3D simulation," Materials Today: Proceedings, vol. 5, no. 9, pp. 19410-19417, 2018.

[11] J. G. Rice and R. J. Schnipke, "An equal-order velocitypressure formulation that does not exhibit spurious pressure modes," Computer Methods in Applied Mechanics and Engineering, vol. 58, no. 2, pp. 135-149, 1986.

[12] H. Zhou, T. Geng, and D. Li, "Numerical filling simulation of injection molding based on 3D finite element model," Journal of Reinforced Plastics and Composites, vol. 24, no. 8, pp. 823-830, 2005.

[13] G. Tie, L. Dequn, and Z. Huamin, “Three-dimensional finite element method for the filling simulation of injection molding," Engineering with Computer, vol. 21, no. 4, pp. 289-295, 2006.

[14] Z. Tadmor, E. Broyer, and C. Gutfinger, "Flow analysis network (FAN)-A method for solving flow problems in polymer processing," Polymer Engineering and Science, vol. 14, no. 9, pp. 660-665, 1974.

[15] H. L. Sheng and F. Liu, Injection Molding Simulation Examples of Moldflow, Publishing House of Electronics Industry, Beijing, China, 2014. 